\title{
Ueber ein eigenthümliches optisches Verhalten der quergestreiften Muskelfaser.
}

\author{
Von
}

Dr. C. L. Meppmer aus St. Petersburg.

(Aus dem Institute für experimentelle Pathologie in Wien.)

Hierzu Taf. IX und ein Holzschnitt.

Seit geraumer Zeit damit beschäftigt, in dem Laboratorium des Herrn Professor Stricker die sichtbaren Veränderungen der quergestreiften Muskelfaser während ihrer Contraction zu studiren, wurde meine Aufmerksamkeit durch die neuerdings publicirten Forschungen auf die rein morphologische Seite dieser Frage gelenkt.

Die Arbeiten von W. Krause und V. Hensen haben in neuester Zeit sowohl für die Structur als Function der quergesreiften Muskelfaser ganz neue Gesichtspunkte aufgestellt. Auf Grund zahlreicher Controlversuche bin ich gezwungen, den von diesen Autoren ausgesprochenen Meinungen entgegenzutreten und halte es daher für nöthig, das Wichtigste dessen, was Krause und Hensen gesagt haben, als die Thesen, welche ich zu widerlegen habe, in folgendem mitzutheilen.

Nach Krause (Ueber den Bau der quergestreiften Muskelfaser. Zeitschr. f. rat. Med. 1868 S. 265-270) soll man bei der Flächenansicht in der Muskelfaser helle, flüssige isotrope Querbänder, die durch $\mathrm{Querlinien} \mathrm{halbirt} \mathrm{werden} \mathrm{und} \mathrm{d} u \mathrm{nkle}$, feste, anisotrope Querbänder zu Gesicht bekommen. Auf dem Querschnitte erblickt man ein Netz von Linien, die sich ganz wie die Querlinien verhalten. Aus diesen Befunden zieht Krause folgende auf die Morphologie des Muskelbündels Bezug habende Schlüsse; Die Querlinien sind 
Membranen, die das Muskelrohr der Quere nach in Muske]fächer abtheilen und für je zwei aneinander gränzende Flächen derselben nur einfach vorhanden sind. Der Länge nach wird der Muskelschlauch hingegen von einem Röhrensystem durchsetzt, dessen Anordnung in den auf dem Querschnitte sichtbaren netzförmigen Linien ihren Ausdruck findet. Die Hüllen der einzelnen Röhren sind an den Contactflächen nicht gemeinschaftlich, wie das bei den als Querlinien zur Ansicht gelangenden Membranen der Fall ist, sondern es besitzt jedes Röhrchen seine selbstständigen Wandungen, die an den Contactflächen von ihren Nachbarn durch die interstitielle Flüssigkeit und durch Fetttröpfchen geschieden werden. Durch das Ineinandergreifen der queren Muskelfächen und des longitudinalen Röhrensystems wird die Muskelfaser in eine Menge wie Bienenwaben über einander gereihte Muskelkästchen zerlegt, von denen jedes eine mit seinem obern oder untern Nachbarn gemeinschaftliche Grundmembran (als Theil der Querlinie) und mehrere selbstständige Seitenmembranen besitzt. Der Inhalt eines Muskelkästchens besteht aus dem festen, dasselbe nicht ganz ausfüllenden Muskelprisma und einer im oberen und untern Abschnitte des Kästchens befindlichen Flüssigkeit, der Muskelkästchenflüssigkeit. Bei der Contraction sollen die Muskelprismen sich mit ihren Basen einander nähern und die Muskelkästchenflüssigkeit von den Grund- zu den Seitenflächen des Muskelkästchens ausweichen.

Hensen (Ueber ein neues Strukturverhältniss der quergestreiften Muskelfaser. Arbeiten des Kieler physiologischen Instituts 1868 S. 1 bis 26) sah an Muskeln sowohl von Wirbelthieren als von Wirbellosen eine Theilung der contractilen Substanz (Quers chei be), durch eine bald helle, bald dunkle feinkörnige Linie (M i t te lscheibe) in zwei Hälften. Die so getheilten Querscheiben werden durch die schwach lichtbrechende für gewöhnlich hell bei Schrägstellung des Spiegels aber dunkel erscheinende Zwischensubstanz von einander geschieden. Bei der Untersuchung im polarisirten Licht erscheint die Querscheibe doppeltbrechend, die Mittelscheibe dagegen einfach lichtbrechend.

Was die biologischen Deductionen betrifft, so stellt sich Hensen den Muskel als einen Apparat vor, der nach den Principien des Electromagnetismus aus kleinen, sich anziehenden Partikeln zusammengesetzt ist. Letztere sollen die Querscheibe zusammensetzen und durch eine, bei der gegenseitigen Attraktion stattfindenden Com- 
pression der elastischen Zwischensubstanz, Verkürzung der ganzen Muskelfaser hervorbringen.

Wenn wir uns vor Allem fragen, welche eigentlich die neuen Entdeckungen sind, auf welche sich $\mathrm{K}$ ra us e und Hensen stützen, so lässt sich darauf vorerst schwer eine direkte Antwort geben; denn wenn wir den Angaben dieser Autoren folgen, begeben wir uns eines ausgezeichneten Hilfsmittels der Verständigung, das ist der Unterscheidung zwischen einer einfach-brechenden Zwischensubstanz und einer doppelt-brechenden contractilen Substanz, welche Hensen wie $\mathrm{Kra}$ use in eigenthümlicher Weise untereinauder geworfen haben. - Wir wollen daher die Objecte selbst zu Rathe ziehen und von der Beobachtung geleitet, den neuen Anschauungen folgen. Die Darstelluug bezieht sich ausschliesslich auf die lebenden Muskelfasern des Hydrophilus piceus. Ich habe auch die Muskeln mehrerer Wirbelthiere (Mensch, Hund, Katze, Ratte, Frosch etc.), sowie die des Flusskrebses frisch und mit Spiritus, Säuren oder Salzlösungen behandelt, untersucht und mich überzeugt, dass sie für diesen einen Zweck nicht mehr lehren als die zuerst genannten.

Die frische, nicht gequetschte und ruhende Faser des Hydrophilus-Muskels ist ein cylindrischer Strang, an dem man ohne Zusatz von Reagentien bekanntlich eine scharf markirte Theilung in querliegende Zonen wahrnimmt. Bei medianer oder $45^{\circ}$ gegen den $\mathrm{Ho}$ rizont geneigter Spiegelstellung bemerkt man je eine breite, matt hell aussehende Zone (Taf. IX a) auf die zu beiden Seiten eine schmälere, glänzende und hellere Zone (b) folgt; an diese reiht sich jederseits eine Linie (c), welche bei schwacher Vergrösselung als ein gleichmässiger dunkler Streifen, bei starker (800-1000) aber granulirt erscheint. Aus dem Aufbau der $\mathrm{M}$ uskelfaser aus diesen drei, vorläufig nur durch ihre verschiedene Lichtstärke unterschiedenen, Zonen, leuchtet ein, dass auch der granulirte Streifen jederseits von einer hellen glänzenden Zone begrenzt ist. Diese beiden, eine dunkle Linie einschliessenden glänzenden Zonen will ich, vorläufig He nsen folgend, zusammenfassen und kurzweg das glänzende Band nennen. - Die Contourirung dieses glänzenden Bandes ist in den meisten Fällen so scharf oder kann bei geeigneter Einstellung des Mikroskopes so scharf gezeichnet werden, dass es in der That nicht leicht wird, sich von dem Gedanken loszusagen, man habe es hier mit einem wirklichen Gebilde $z u$ thun. Ist man aber so glücklich, eine Muskelfaser zur Ansicht zu bekommen, welche diametral durch das 
Sehfeld des Mikroscopes läuft, so wird man jedesmal finden, dass, bei sich gleich bleibender Einstellung, nie alle glänzenden Bänder, die man übersehen kann, ein gleiches Verhältniss zu den sie halbirenden dunklen Streifen haben. Hat man z. B. den in der Mitte des Sehfeldes liegenden Abschnitt der Faser so eingestellt, dass das glänzende Band von dem körnigen Streifen halbirt wird, so erscheinen die gegen den einen oder den andern Rand des Sehfeldes liegenden Zeichnungen so angeordnet, dass die dunkle Linie allmählig ihre Lagerung im glänzenden Bande ändert, d. h. sich gegen den einen oder andern Rand desselben hinbegiebt (Vergl. Taf. IX). Nimmt man die Abstände je zweier dunkler Streifen ins Auge, so erfährt man bald, dass sich diese nicht ändern, sondern dass die glänzenden Querbänder es sind, welche die Verschiebung erleiden.

Durch Veränderung der Einstellung kann das Verschieben des glänzenden Bandes so sehr gesteigert werden, dass es fast bis zur Mitte der matthellen Substanz gebracht werden kann, wobei es jedoch seine scharfe Contourirung einbüsst. Man kann ferner Stellen hervorbringen, wo das glänzende Band gänzlich fehlt und nur matthelle Zonen mit granulirten Streifen abwechseln (Taf. IX $\mathbf{a}^{\prime} . \mathrm{c}^{\prime}$.), kurz wo man im Sinne Rolletts (Untersuchung zur näheren Kenntniss des Baues der quergestreiften Muskelfaser. Sitzungsber. der Wiener Acad. d. Wissensch. Bd. 24. 1857. S. 292) breite Hauptsubstanz und schmälere $Z$ wischensubstanz-S cheiben mit einander wechseln sieht. Noch weit auffallender als durch die veränderte Einstellung wird das Phänomen der Wanderung des glänzenden Bandes durch Aenderung der Spiegelstellung zur Anschauung gebracht. Um frappante Bilder zu erhalten, Yeistet ein Planspiegel bessere Dienste als ein concaver und dann eignet sich Lampenlicht besser dazu als diffuses Tageslicht. Die Ortsveränderung des lichten Bandes tritt sowohl bei Drehung des Spiegels um die laterale als sagittale Horizontalaxe ein. Am auffallendsten werden aber die Wandererscheinungen des glänzenden Bandes wahrgenommen, wenn man sein Präparat durch ein Mikroskop mit drehbarem Tisch untersucht. Je nachdem die Querstreifung des Muskelbündels ihre Stellung zur Beleuchtung wechselt, ändert sich auch das Ansehen und Verhältniss des glänzenden Bandes in der so eben angegebenen Weise.

An dem Querschnitt des lebenden Hydrophilusmuskels fällt ein ähnliches Verhalten, wie bei der Flächenansicht auf.

$\mathrm{Da}$, wo an den Grenzen der einzelnen Sarcons elements fein- 
granulirte schmale Linien zu sehen sind, erscheinen neben diesen auch glänzende Streifen, die alle genannten Eigenschaften der glänzenden Bänder aufweisen. Bei dem oben angegebenen Verfahren der Spiegelwendung und Drehung des Tisches sieht man diese Linien sich verschieben, an einem Rande eines Präparats verschwinden, um an einem andern wieder aufzutauchen.

Aus dem Auseinandergesetzten ist es sofort klar, dass man bei verschiedenen Spiegelstellungen, bei verschiedenen relativen Lagen von Object und Spiegel überhaupt, bald Bilder sieht, welche bei der Flächenansicht der Muskelfaser auf ein einfaches Alterniren von hellen und dunklen, ungleich breiten Scheiben, und bald wieder auf eine Complication schliessen lassen, wie sie Hensen und Krause gesehen und in so verschiedener Weise gedeutet haben. Dass nicht alle Bilder auf anatomischen Grundlagen ruhen, braucht nicht erwiesen zu werden. Der Spiegel kann den Gang der Lichtstrahlen, nicht aber den Bau des Muskels beeinflussen. Welches Bilıl ist nun das richtige? Welches, mit andern Worten, hat eine morphologische Grundlage?

Zunächst werden wir annehmen müssen, dass der dunkle Streifen Ausdruck eines wirklichen Gebildes ist, da er bei allen Relationen zwischen Spiegel und Object unverändert bleibt. Dabei soll vorläufig davon abstrahirt werden, was die Verschiedenheit der Einstellung bewirkt. Wir gehen von einer und zwar von der obersten Mantelfläche einer ruhenden Faser aus, und hier sehen wir, dass die körnigen Streifen relativ dunkel bleiben, man mag den Spiegel stellen wie man wolle.

Sucht man die Faser zwischen gekreuzten Nicols auf, so stimmt nur dieser Streifen mit dem Gesichtsfelde überein; er ist dunkel, wenn das Gesichtsfeld dunkel ist und nimmt dessen Farbe an, wenn es durch eine Glimmerplatte gefärbt wird.

Die granulirten Streifen sind also einfach-brechend. Alles, was zwischen ihnen liegt, erscheint bei gekreuzten Nicols hell, ist also doppelt-brechend. Doch ist auch hier eine Verschiedenheit in der Lichtintensität zu constatiren, da auch im polarisirten Lichte glänzende Bänder gegen mattere aber noch helle Zonen abgesetzt erscheinen.

Recapituliren wir das Gesagte in Kürze, so ist also ein einfachbrechender Streifen, der sich im gemeinen Lichte dunkler als seine Umgebung darstellt, das eine Stück, welches wir festhalten müssen, und, wie die Sachen liegen, festhalten können, da dieser Streifen ja 
mit der Querlinie Krauses, und mit der Mittelscheibe Hensens identisch ist. Diesem Streifen liegen helle, glänzende Bänder zur Seite, die bei wechselnder Spiegelstellung ihre Breite ändern und zwar von der Null aufwärts zu einer nicht gemessenen Breite. Auf diese glänzenden Bänder folgen die matten Zonen, deren Helligkeit die Mitte hält zwischen den dunkeln Streifen und den glänzenden Bändern. Die Breite auch dieser matten Zonen wechselt bei veränderter Spiegelstellung, aber sie wird bei dem nicht contrahirten Muskel niemals Null. Die glänzenden Bänder allein sind es also, welche beigewisser Spiegelstellung schwinden.

Wenn Jemand mit der Behauptung aufträte, diese glänzenden Bänder seien lediglich der Ausdruck totaler Reflexion, sie schwinden, wenn die Incidenz des Lichtes diese ausschliesst und sie kommen wieder und nehmen an Breite zu, mit der Zunahme des Einfallswinkels, so könnte man ihn nicht stichhaltig widerlegen. - Denn

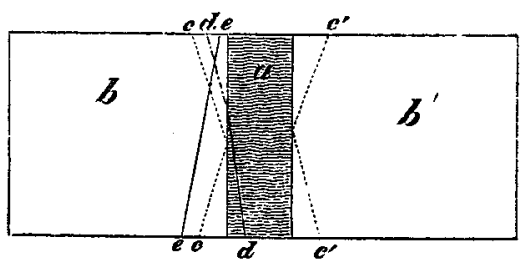
denken wir uns in a eine Substanz von kleinerem und in $b$ und $b^{\prime}$ je eine von grösserem Brechungsindex, in welche die Strahlen $c$ und $c^{\prime}$ mit hinreichend grossem Einfallswinkel von unten her eindringen, um an der Grenze von $b$ und a total reflectirt zu werden, dann muss an jeder Seite von a eine Zone von b liegen, welche heller als der Rest erscheint, denn diese Zone wird mehrfach beleuchtet, nänlich von den einfach gebrochenen Strahlen e, den total reflectirten $\mathrm{c}$ und von den aus $a$ nach $b$ gebrochenen Strahlen. Mit derselben Behauptung könnte man das Verschwinden und die wechselnde Breite vertheidigen, denn die Annahme der Verschiedenheit des Brechungsindex festgehalten, muss ja dieser Wechsel eintreten mit dem Wechsel der Richtung der Lichtstrahlen.

Der Versuch mit der Aenderung in der Neigung des Spiegels und der Drehung des Tisches ist so präcise, dass man aus diesem allein auf einen Unterschied in den Brechungsindices der beiden Substanzen schliesen könnte, und er ist wohl schon ein Beweis für die oben hypothetisch angeführte Behauptung. Gehen wir aber einen Schritt weiter, indem wir künstlich die Differenz der Brechungsindices der beiden Muskelsubstanzen ändern. Es ist das ein in der mikroskopischen Technik sehr gebräuchliches Verfahren. Lassen 
wir ein Reagens zufliessen, welches die Muskelfaser gleichmässig infiltrirt, wie z. B. Alkohol, so ändert sich das Bild. Die glänzenden Bänder schwinden entweder gänzlich oder erblassen wenigstens ganz bedeutend und wieder haben wir nur breite matthelle Zonen zwischen dunkeln, schmalen, feinkörnigen Streifen. Indem beide Substanzen von Alkohol durchtränkt werden, müssen die Brechungsindices beider gegen den des Alkohols hin geändert, die Differenz somit verkleinert werden und es ist von selbst klar, warum jetzt die totale Reflexion nicht mehr in dem Grade wahrzunehmen ist, wie früber.

Ich habe schon erwähnt, dass Alles, was zwischen den einfach lichtbrechenden Streifen liegt, im gekreuzten Nicol hell erscheint. Nur ist auch hier ein matterer und ein hellerer, glänzenderer Theil $\mathrm{zu}$ unterscheiden. Ich weiss nicht, mit welchen optischen Hilfsmitteln man es noch erklären könnte, dass diese zwei Zonen, welche beide doppeltbrechend sein müssen, im gekreuzten Nicol verschiedene Helligkeit bieten. Zweifellos reichen die Vorgänge bei der totalen Reflexion hin, um Helligkeits-Unterschiede zu erklären.

Wir können also die Muskelfaser nicht anders ansehen, als sie B r ü cke (Untersuchungen über den Bau der Muskelfasern mit Hülfe des polarisirten Lichtes. Denksch. d. Kais. Acad. d. Wissensch. in Wien. Bd. XV. S. 69-84. Taf. 1) gezeichnet hat: Sie ist im ruhenden Zustande zusammengesetzt aus einfachbrechenden schmalen und aus einer breiten doppeltbrechenden, oder, um mit Rollett zu sprechen, aus einer Zwischensubstanz von geringerem und einer Hauptsubstanz von grösserem Brechungsindex.

Die Mittelscheibe Hensen's ist, sowie die Querlinie Krause's nichts anderes als die schwächer lichtbrechende isotrope Zwischensubstanz, die Querscheibe Hensen's (l. c., vergl. Fig. 4. 5. 6., sowie S. 25) sowie die Muskelkästchenflüssigkeit $\mathrm{Kr}$ a u se's sind Produkte der Spiegelung. Das Muskelprisma Krause's hat Hensen als Zwischensubstanz bezeichnet und es ist von selbst klar, dass hier jene Abschnitte der contractilen Substanz gemeint sind, welche nicht in den Bereich der totalen Reflexion fallen.

Die Bilder, auf welche Krause und Hensen, meines Wissens zuerst, hingewiesen haben, sind also in Wirklichkeit $z \mathrm{u}$ sehen. Die Beobachtungen dieser beiden Forscher haben auch unsere Kenntnisse bereichert; denn sie haben uns gelehrt, dass an den quergestreiften Muskelfasern mehr zu sehen ist, als das bekannte Schema 
144 C.L.Hep pner: Ueb. ein eigenthüml. Verhalt. d. quergestreiften Muskelfaser.

verträgt. Die Beobachtung beider Autoren, stimmt auch darin überein, dass beide eine von einer dunklen Scheidewand getheilte Zone beschrieben haben. Die Differenz zwischen den Auffassungen beider liegt, soweit es die Beobachtung betrifft, nur darin, dass Hensen dasjenige Zwischensubstanz nennt, was Krause als den wichtigsten Bestandtheil, als Muskelprisma hinstellt. In der That liegt die Wahrheit zwischen beiden Angaben; das Muskelprisma sammt der Muskelkästchenflüssigkeit $\mathrm{Krause}$ 's, die Zwischensubstanz und die Querscheiben Hensen's, sind nur auf Grundlage der Spiegelung auseinandergehalten: sie gehörten in toto der Hauptsubstanz an, sind in toto doppeltbrechend und von grösserem Brechungsindex als die dünne Zwischensubstanz-Scheibe.

\section{Erklärung der Abbildung auf Taf. IX.}

Muskelfaser von Hydrophylus piceus im ruhenden Zustande mit Immersionslinse Nr. 10 und Oc. Nr. 3 Hartnack auf circa $36 \mathrm{Cm}$. Distanz gezeichnet.

c. Zwischensubstanz.

a. und b. Hauptsubstanz. 


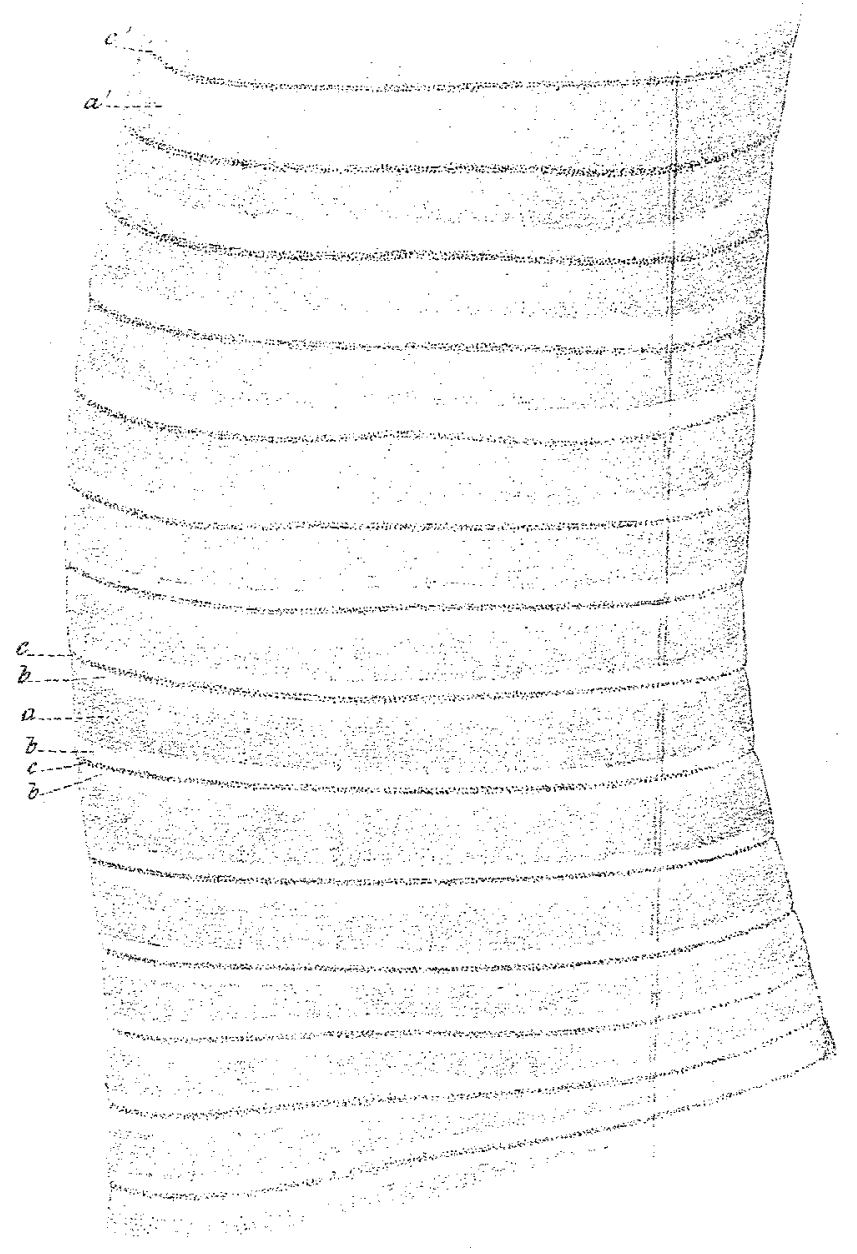

